\title{
Transmission of Optical Communication Signals by Distributed Parametric Amplification
}

\author{
Georgios Kalogerakis, Student Member, IEEE, Michel E. Marhic, Senior Member, IEEE, Member, OSA, \\ Kenneth Kin-Yip Wong, Member, IEEE, and Leonid G. Kazovsky, Fellow, IEEE, Fellow, OSA
}

\begin{abstract}
For the first time, to the authors' knowledge, distributed parametric amplification (DPA), i.e., the use of a transmission fiber itself for parametric amplification of communication signals is proposed and demonstrated. To account for the inevitable fiber loss, solutions were derived for the distributed amplifier, with either one or two pumps: They are obtained in terms of confluent hypergeometric functions. Low-penalty DPA of a 10-Gb/s nonreturn-to-zero (NRZ) signal over a 75-km dispersionshifted fiber (DSF), is demonstrated by using only $66.5 \mathrm{~mW}$ of pump power. Three adjacent channels have been simultaneously transmitted, with little penalty due to nonlinear crosstalk. It is experimentally verified that DPA requires less pump power than distributed Raman amplification (DRA), for similar power penalties.
\end{abstract}

Index Terms-Distributed amplification, optical amplifier, optical parametric amplifier (OPA).

\section{INTRODUCTION}

D URING the past decade, a great deal of research has been performed for developing fiber Raman amplifiers. Some work has been done on discrete Raman amplifiers, but it appears that the best way to utilize Raman gain is for distributed amplification, i.e., to amplify communication signals along the long transmission fibers themselves, rather than in discrete amplifiers located between transmission fibers. A number of system manufacturers have developed next-generation longhaul systems based at least in part on distributed Raman amplification (DRA). System operators, however, remain cautious about such systems, as they generally require high pump powers, sometimes in excess of $1 \mathrm{~W}$, which raise concerns about safety and reliability.

In recent years, there has also been considerable interest in fiber optical parametric amplifiers (OPAs), as their versatility and performance make them attractive for possible application in areas such as optical communication and high-power wavelength conversion (for a recent review of the state of the art, see, for example, [1]). To date, essentially all efforts have concentrated on discrete fiber OPAs, i.e., subsystems that could eventually be placed in a box and used as a substitute for other discrete optical amplifiers, such as erbium-doped fiber amplifiers (EDFAs).

Manuscript received November 30, 2004; revised June 8, 2005. This work was supported in part by National Science Foundation Grant ANI-0123441.

G. Kalogerakis, M. E. Marhic, and L. G. Kazovsky are with the Electrical Engineering Department, Stanford University, Stanford, CA 94305 USA (e-mail: gkalog@stanford.edu).

K. K.-Y. Wong is with the Electrical and Electronic Engineering Department, The University of Hong Kong, Pokfulam, Hong Kong.

Digital Object Identifier 10.1109/JLT.2005.855668
Raman and parametric fiber amplification are related thirdorder nonlinear phenomena, which both occur in common silica fibers in the presence of strong optical pumps. Whether a particular gain mechanism is best suited for discrete or distributed amplification depends on a number of characteristics, such as gain bandwidth, required pump power, and noise characteristics.

Distributed parametric amplification (DPA) has been investigated in the past as a detrimental effect, as strong carriers can amplify in transmission fibers the amplified spontaneous emission (ASE) generated by discrete EDFAs used to amplify signals between fiber spans. This has been done for onepump [2]-[4] and two-pump [5] parametric amplification. By contrast, this paper, for the first time to the authors' knowledge investigates a possibly beneficial application of DPA, namely, amplification of communication signals along a transmission fiber by using a copropagating pump.

Compared to DRA, DPA has the following advantages.

1) Pump power: DPA requires less pump power than DRA to obtain the same gain. This is attractive, as the power levels required for DRA have raised safety and reliability concerns.

2) Double Rayleigh scattering (DRS): Because parametric gain in fibers occurs only for copropagating pump(s) and signals, DPA is free from DRS, which is a significant problem for DRA.

3) Pump attenuation: DPA pumps can have about the same wavelength as the signals; hence, pumps and signals have about the same loss. By contrast, in DRA, pumps necessarily have wavelengths about $100 \mathrm{~nm}$ shorter than the signals, and therefore, the pumps have higher loss than the signals.

4) Idler generation: In DPA, amplification of the signal is accompanied by the generation of an idler, which is not present in DRA. The idler has several important properties that make it potentially interesting for improving signal-to-noise (SNR) ratio, etc.

In spite of the long transmission spans (generally $>40 \mathrm{~km}$ ), the pump power $P_{0}$ required to make up for fiber loss is relatively modest, of the order of $100 \mathrm{~mW}$. This implies that the gain bandwidth will be fairly small, at best of the order of $10 \mathrm{~nm}$. Hence, DPA will not compete with methods providing much larger bandwidths, such as DRA, tellurite EDFAs, etc. On the other hand, DPA may be a useful supplement for these other techniques: For example, it could be used for transmission near $1300 \mathrm{~nm}$ in a system using standard single-mode 
fiber (SMF) primarily for transmission in the $C$ band. DPA could also potentially be used for coarse-wavelength-divisionmultiplexing (CWDM), by using several pumps, separated by tens of nanometers.

For these reasons, we have undertaken a preliminary investigation of DPA and report the results here. The paper is organized as follows. In Section II, a closed-form solution is derived for the gain of fiber OPAs in the presence of fiber loss, which cannot be ignored in DPA. The solution is obtained in terms of confluent hypergeometric functions; it is useful for estimating required pump power, gain bandwidth, etc. Section III describes experiments performed in a 75-km-long dispersion-shifted fiber (DSF) transmission link. By using a single pump, with the remarkably low power of $66.5 \mathrm{~mW}$, low-penalty transmission of a $10-\mathrm{Gb} / \mathrm{s}$ signal near $1550 \mathrm{~nm}$ has been obtained. We have also simultaneously transmitted three adjacent channels, with little degradation due to nonlinear crosstalk. Section IV describes the results of an experimental comparison between DPA and DRA, confirming the pump-power advantage of DPA. In Section V, results and potential implications for communication systems are discussed and conclusions are given.

\section{THEORY OF DPA IN LOSSY FIBERS}

Since the fiber loss in DPA is fairly large (e.g., about $15 \mathrm{~dB}$ for $80 \mathrm{~km}$ of DSF), the common expressions for OPA gain, obtained by neglecting fiber loss, are inapplicable. One must then go back to first principles to derive the gain expressions applicable in the case of lossy fibers. Here, we present a derivation, which provides the solution for either one- or two-pump OPAs.

The basic equations for one- and two-pump fiber OPAs are obtained by considering four-wave mixing (FWM) interactions among three or four waves, respectively. We assume that the pump(s) are not depleted by the interaction but only suffer exponential attenuation due the distributed loss. The initial system of equations then reduces to two coupled equations for the signal and the idler. Eliminating one unknown then leads to a second-order ordinary differential equation (ODE), which can be solved in terms of confluent hypergeometric functions.

We assume that the fiber nonlinearity and attenuation coefficients $\gamma$ and $\alpha$ are frequency independent. Then, for either oneor two-pump OPAs, the FWM equations for the signal $(k=3)$ and the idler $(k=4)$ are of the form [6]

$\frac{\mathrm{d} A_{k}}{\mathrm{~d} z}=-p A_{k}+r \exp \left(\int_{\xi=0}^{z} q \mathrm{~d} \xi\right) A_{l}^{*}, \quad k=3,4, \quad l=7-k$

where $A_{k}$ represents the complex slowly-varying envelopes, $z$ is distance along the fiber, and ${ }^{*}$ indicates complex conjugation. $p, q$, and $r$ are given by

$$
\begin{aligned}
& p=\frac{\alpha}{2}-2 i \gamma P_{0} \mathrm{e}^{-\alpha z} \\
& q=-\alpha-i \Delta \beta+\left(1+n_{\mathrm{p}}\right) i \gamma P_{0} \mathrm{e}^{-\alpha z} \\
& r=2 i \gamma \sqrt{P_{10} P_{20}}
\end{aligned}
$$

where $P_{10}$ and $P_{20}$ are the initial pump powers; $P_{0}=P_{10}+$ $P_{20} ; n_{\mathrm{p}}$ is the number of pumps; and $\Delta \beta$ is the wavevector mismatch. These expressions are applicable for either one- or two-pump OPAs, provided that the convention $P_{10}=P_{20}=$ $P_{0} / 2$ is used for one-pump OPAs.

Upon letting

$$
B_{k}=A_{k} \exp \left(\int_{\xi=0}^{z} p \mathrm{~d} \xi\right)
$$

we obtain

$\frac{\mathrm{d} B_{k}}{\mathrm{~d} z}=r \exp \left(-\int_{\xi=0}^{z} s \mathrm{~d} \xi\right) B_{l}^{*}, \quad$ where $s=p^{*}-p-q$.

Letting $B_{k}=C_{k} \exp \left(-(1 / 2) \int_{\xi=0}^{z} s \mathrm{~d} \xi\right)$, and eliminating $C_{l}$ leads to $\mathrm{d}^{2} C_{k} / \mathrm{d} z^{2}-g^{2}(z) C_{k}=0$, where $g^{2}(z)=|r|^{2}$ $\exp (-2 \alpha z)+s^{2} / 4+s^{\prime} / 2$, and prime stands for a derivative with respect to $z$. It is found that $g^{2}(z)$ is of the form $g^{2}(z)=$ $u \exp (-2 \alpha z)+v \exp (-\alpha z)+w$, where

$$
\begin{aligned}
& u=\gamma^{2}\left[4 P_{10} P_{20}-\frac{\left(3-n_{\mathrm{p}}\right)^{2} P_{0}^{2}}{4}\right] \\
& v=-\frac{\left(3-n_{\mathrm{p}}\right) \Delta \beta \gamma P_{0}}{2} \\
& w=\frac{(\alpha+i \Delta \beta)^{2}}{4} .
\end{aligned}
$$

The initial conditions for $C_{k}$ are $C_{k}(0)=A_{k}(0)$, and $C_{k}^{\prime}(0)=s(0) A_{k}(0) / 2+r A_{l}^{\prime}(0)$.

Letting $x=\exp \{-\alpha z\}$, we obtain

$\frac{\mathrm{d}^{2} C_{k}}{\mathrm{~d} x^{2}}+\frac{1}{x} \frac{\mathrm{d} C_{k}}{\mathrm{~d} x}-\left(\frac{u}{\alpha^{2}}+\frac{v}{\alpha^{2} x}+\frac{w}{\alpha^{2} x^{2}}\right) C_{k}=0, \quad k=3,4$.

Letting $C_{k}=Y_{k} \sqrt{x}$ yields

$$
\frac{\mathrm{d}^{2} Y_{k}}{\mathrm{~d} x^{2}}+\left[-\frac{u}{\alpha^{2}}-\frac{v}{\alpha^{2} x}+\left(\frac{1}{4}-\frac{w}{\alpha^{2}}\right) \frac{1}{x^{2}}\right] Y_{k}=0 .
$$

Letting $x=Z / Z_{0}$, where $Z_{0}=Z(0)$ is a constant, yields

$$
\frac{\mathrm{d}^{2} Y_{k}}{\mathrm{~d} Z^{2}}+\left[-\frac{u}{\alpha^{2} Z_{0}^{2}}-\frac{v}{\alpha^{2} Z_{0} Z}+\left(\frac{1}{4}-\frac{w}{\alpha^{2}}\right) \frac{1}{Z^{2}}\right] Y_{k}=0
$$

Letting

$$
Z_{0}=\frac{2 \sqrt{u}}{\alpha}, \quad \kappa=-\frac{v}{\alpha^{2} Z_{0}}, \quad \mu=\frac{w^{\frac{1}{2}}}{\alpha}
$$

we obtain

$$
\frac{\mathrm{d}^{2} Y_{k}}{\mathrm{~d} Z^{2}}+\left[-\frac{1}{4}+\frac{\kappa}{Z}+\left(\frac{1}{4}-\mu^{2}\right) \frac{1}{Z^{2}}\right] Y_{k}=0
$$

which is Whittaker's equation [7]. 
Two independent solutions are the Whittaker confluent hypergeometric functions $M_{\kappa, \mu}(Z)$ and $W_{\kappa, \mu}(Z)$. We must then have $Y_{k}(Z)=U_{k} M_{\kappa, \mu}(Z)+V_{k} W_{\kappa, \mu}(Z)$, where $U_{k}$ and $V_{k}$ are constants to be determined from the initial conditions, which are $Y_{k}\left(Z_{0}\right)=C_{k}(0)$ and $Y_{k}^{\prime}\left(Z_{0}\right)=\left(1 / Z_{0}\right)$ $\left[(1 / 2) C_{k}(0)-(1 / \alpha) C_{k}^{\prime}(0)\right]$.

The solution for the idler field $(k=4)$ is relatively simple if no idler is present at the input. Then, the equations for $U_{4}$ and $V_{4}$ are

$$
\begin{aligned}
& Y_{4}\left(Z_{0}\right)=U_{4} M_{\kappa, \mu}\left(Z_{0}\right)+V_{4} W_{\kappa, \mu}\left(Z_{0}\right)=0 \\
& Y_{4}^{\prime}\left(Z_{0}\right)=U_{4} M_{\kappa, \mu}^{\prime}\left(Z_{0}\right)+V_{4} W_{\kappa, \mu}^{\prime}\left(Z_{0}\right)=-\frac{r}{\sqrt{u}} A_{3}^{*}(0) .
\end{aligned}
$$

The solution is $U_{4}=(r / 2 \sqrt{u}) A_{3}^{*}(0)\left[W_{\kappa, \mu}\left(Z_{0}\right) / W_{\text {Whit }}\left(Z_{0}\right)\right]$ and $V_{4}=-(r / 2 \sqrt{u}) A_{3}^{*}(0)\left[M_{\kappa, \mu}\left(Z_{0}\right) / W_{\text {Whit }}\left(Z_{0}\right)\right]$, where $W_{\text {Whit }}\left(Z_{0}\right)$ is the Wronskian of the Whittaker functions $M_{\kappa, \mu}(Z)$ and $W_{\kappa, \mu}(Z)$ at $Z_{0}$, i.e., $W_{\text {Whit }}\left(Z_{0}\right)=M_{\kappa, \mu}\left(Z_{0}\right)$ $W_{\kappa, \mu}^{\prime}\left(Z_{0}\right)-W_{\kappa, \mu}\left(Z_{0}\right) M_{\kappa, \mu}^{\prime}\left(Z_{0}\right)$. It is shown in Appendix A that $W_{\text {Whit }}\left(Z_{0}\right)=-\Gamma(b) / \Gamma(a)$. We can then write

$$
\begin{aligned}
Y_{4}(Z)=\frac{r}{\alpha Z_{0}} \frac{\Gamma(a)}{\Gamma(b)}\left[W_{\kappa, \mu}(Z) M_{\kappa, \mu}\left(Z_{0}\right)\right. & \\
& \left.-W_{\kappa, \mu}\left(Z_{0}\right) M_{\kappa, \mu}(Z)\right] A_{3}^{*}(0) .
\end{aligned}
$$

The idler power gain (or conversion efficiency) can then be calculated as

$$
\begin{aligned}
G_{\mathrm{i}}(z)= & \left|\frac{A_{4}(z)}{A_{3}^{*}(0)}\right|^{2} \\
= & \mathrm{e}^{-\alpha z}\left|\frac{Y_{4}(Z)}{A_{3}^{*}(0)}\right|^{2} \\
= & \mathrm{e}^{-\alpha z} \mid \frac{r}{\alpha Z_{0}} \frac{\Gamma(a)}{\Gamma(b)}\left[W_{\kappa, \mu}(Z) M_{\kappa, \mu}\left(Z_{0}\right)\right. \\
& \left.-W_{\kappa, \mu}\left(Z_{0}\right) M_{\kappa, \mu}(Z)\right]\left.\right|^{2} .
\end{aligned}
$$

An alternate expression can be obtained in terms of the Kummer functions $M$ and $U$ (see Appendix A). It is

$$
\begin{aligned}
G_{\mathrm{i}}(z)=\mathrm{e}^{-\alpha z} \mid \frac{r}{\alpha} & \frac{\Gamma(a)}{\Gamma(b)} \mathrm{e}^{-\frac{\left(Z+Z_{0}+\alpha b z\right)}{2}} Z_{0}^{b-1} \\
\times & {\left[U(a, b, Z) M\left(a, b, Z_{0}\right)\right.} \\
& \left.\quad-U\left(a, b, Z_{0}\right) M(a, b, Z)\right]\left.\right|^{2} .
\end{aligned}
$$

The advantage of this form for $G_{\mathrm{i}}$ is that it is expressed only in terms of $M$ and $U$, which are confluent hypergeometric functions available in mathematical programs such as Mathematica. ${ }^{1}$ By using the relationship between $M$ and $U$, one can also write an expression for $G_{\mathrm{i}}$ entirely in terms of $M$ (this is

${ }^{1}$ http://www.wolfram.com

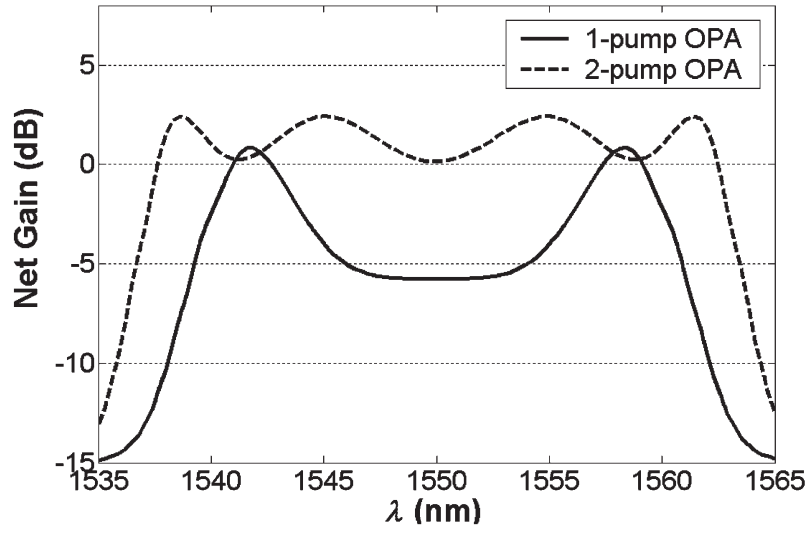

Fig. 1. Theoretical DPA gain spectra for one pump at $\lambda_{1}=\lambda_{0}=1550 \mathrm{~nm}$, and two pumps at $\lambda_{1}=1540.01 \mathrm{~nm}$ and $\lambda_{2}=1560.00 \mathrm{~nm}$, respectively. Total pump power is $66 \mathrm{~mW}$ for both cases.

advantageous for taking certain limits, since the properties of $M$ are better known than those of $U$ ). This yields

$$
\begin{aligned}
& G_{\mathrm{i}}(z)=\mid \frac{\pi r}{\alpha} \frac{\mathrm{e}^{-\frac{\left(Z+Z_{0}\right)}{2}}}{\sin (\pi b) \Gamma(b) \Gamma(d)} \\
& \times\left[M(c, d, Z) M\left(a, b, Z_{0}\right) x^{\frac{3-b}{2}}\right. \\
&\left.\quad-M\left(c, d, Z_{0}\right) M(a, b, Z) x^{\frac{1+b}{2}}\right]\left.\right|^{2}
\end{aligned}
$$

where $c=1+a-b$ and $d=2-b$.

The expression for $A_{3}(z)$ is more complicated than for $A_{4}(z)$. However, the signal power gain $G_{\mathrm{S}}(z)=\left|A_{3}(z)\right|$ $\left.A_{3}(0)\right|^{2}$ can be obtained simply from $G_{\mathrm{i}}(z)$, since it can be shown that $G_{\mathrm{S}}(z)=G_{\mathrm{i}}(z)+\exp (-\alpha z)$ (see Appendix B).

In the case of a one-pump OPA, it can be shown that $u=0$, $Z_{0}=0$, and $\kappa$ is infinite. Then $M(a, b, Z)$ can be expressed in terms of modified Bessel functions $I$ by taking suitable limits [7].

The advantage of these closed-form solutions is that they can be used for rapidly obtaining the shape of the gain spectrum under specified experimental conditions. This permits the rapid optimization in terms of pump power, gain bandwidth, etc.

Fig. 1 shows theoretical gain spectra for one- and twopump DPAs using $75 \mathrm{~km}$ of DSF, with the following parameters: loss $\alpha=0.2 \mathrm{~dB} / \mathrm{km}, \beta_{4}=-5.0 \times 10^{-55} \mathrm{~m}^{-1} \cdot \mathrm{s}^{4}$; zero-dispersion wavelength $\lambda_{0}=1550 \mathrm{~nm}$; dispersion slope $D_{\lambda}=0.07 \mathrm{ps} /(\mathrm{nm} \cdot \mathrm{km})$; nonlinearity coefficient $\gamma=2 \mathrm{~W}^{-1}$. $\mathrm{km}^{-1}$; and total input pump power $P_{0}=66 \mathrm{~mW}$.

The gain spectrum for the two-pump case is interesting, as it is very similar to the Chebyshev gain spectra that can be obtained with lossless fibers [8], even though the total fiber loss here is considerable (about $15 \mathrm{~dB}$ ).

The gain shapes of Fig. 1 are quite sensitive to the choice of the pump wavelength(s); this is particularly true for the twopump OPA case, for which decreasing $\lambda_{1}$ by $0.01 \mathrm{~nm}$ leads to a drop of the center of the spectrum by about $5 \mathrm{~dB}$. This high sensitivity is due to the fact that we have assumed that $\lambda_{0}$ is constant along the fiber. In real fibers, however, $\lambda_{0}$ varies randomly along the fiber length, with a standard deviation $\sigma_{0}$ 

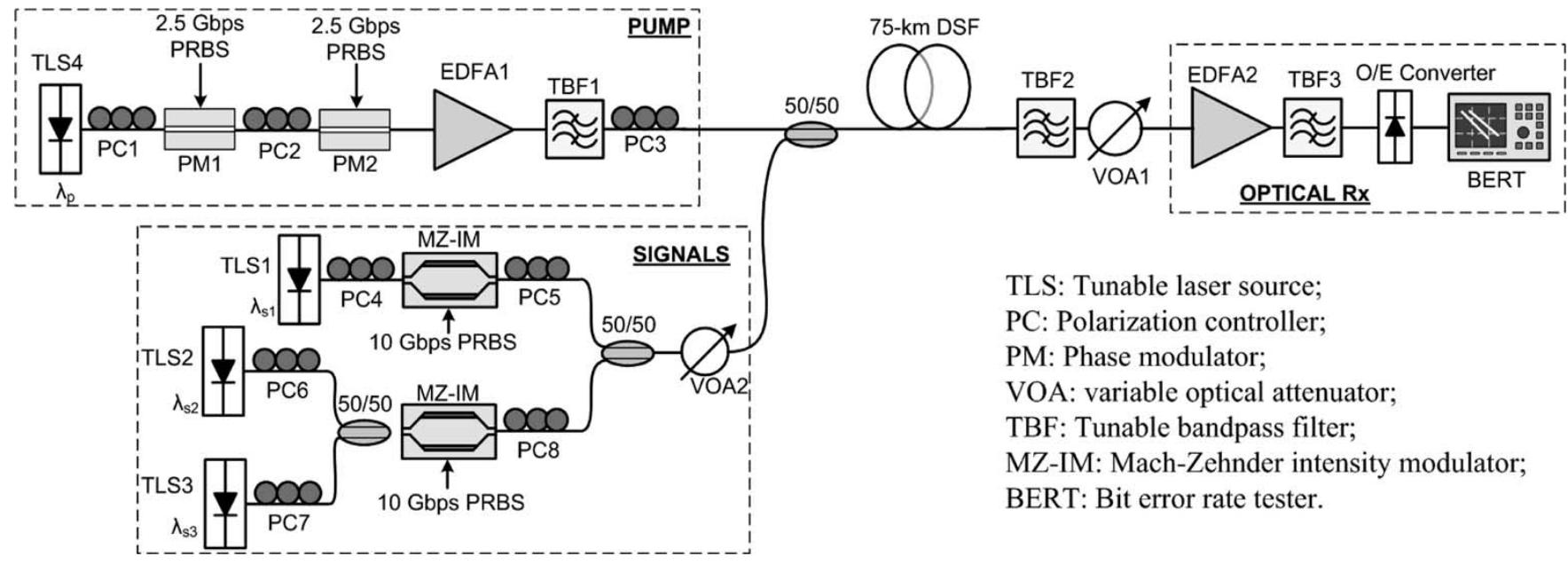

TLS: Tunable laser source;

PC: Polarization controller;

PM: Phase modulator;

VOA: variable optical attenuator;

TBF: Tunable bandpass filter;

MZ-IM: Mach-Zehnder intensity modulator;

BERT: Bit error rate tester.

Fig. 2. Distributed OPA experimental setup.

of the order of $1 \mathrm{~nm}$. Then, it is clear that the gain spectrum cannot be affected by changes in $\lambda_{1}$ much smaller than $\sigma_{0}$. This has been verified experimentally in [5], where it was found that a $2.5-\mathrm{nm}$ change in $\lambda_{1}$ was required in order to obtain a drop in gain of $5 \mathrm{~dB}$. Hence, the gain shape in real fibers, while not exactly predictable by the theory for uniform fibers, should be relatively insensitive to pump wavelength(s). $\lambda_{0}$ variations can also help to obtain a flat-topped gain spectrum, as indicated by the results of [5]. If needed, gain flattening could be implemented by means of a filter at the fiber output. This filter could also remove the residual pump power and the idler(s). Fig. 1 indicates that a signal-gain bandwidth of the order of $10 \mathrm{~nm}$ could be available with either type of DPA with suitable gain flattening. The precise definition of bandwidth is of course tied to system performance, and it could drop to just a few nanometers if the received signal level is too low; in that case, increasing pump power would be required to increase the bandwidth.

\section{DISTRIBUTED OPA EXPERIMENT}

We performed DPA experiments to check whether compensation of transmission fiber loss could indeed be obtained at the pump-power levels predicted by theory and to see whether the achievable gain bandwidths also matched with theory.

The experimental DPA setup is shown in Fig. 2. The transmission fiber consisted of three $25-\mathrm{km}$ DSF spools with average zero-dispersion wavelengths $\lambda_{0}$ of about $1550 \mathrm{~nm}$. The dispersion slope was $0.07 \mathrm{ps} /\left(\mathrm{nm}^{2} \cdot \mathrm{km}\right)$, and $\gamma=2 \mathrm{~W}^{-1} \cdot \mathrm{km}^{-1}$. $\alpha$ was about $0.207 \mathrm{~dB} / \mathrm{km}$, i.e., the fiber had a total loss of $15.53 \mathrm{~dB}$. A tunable laser source TLS4, which was tuned at $\lambda_{\mathrm{p}}=1551.13 \mathrm{~nm}$, served as the pump source. The continuous-wave (CW) pump was phase modulated by two phase modulators in cascade, PM1 and PM2, in order to suppress stimulated Brillouin scattering (SBS). Both of them were driven by a 2.5-Gb/s pseudorandom bit sequence (PRBS) [9].

Polarization controllers PC1 and PC2 aligned the state of polarization (SOP) of the pump with PM1 and PM2, respectively. The pump was then amplified to the desired power level by a $C$ band EDFA (EDFA1) and filtered by a $1.3-\mathrm{nm}$ tunable bandpass filter (TBF1) to reduce the ASE noise due to EDFA1. Three other TLSs, TLS1, TLS2, and TLS3, tuned at $1549.14,1549.46$, and $1549.76 \mathrm{~nm}$, respectively, were used as signal sources. They were modulated by $10-\mathrm{Gb} / \mathrm{s}$ nonreturnto-zero (NRZ) $2^{23}-1$ PRBS as shown in Fig. 2; a single Mach-Zehnder modulator was used for both TLS2 and TLS3, while TLS1 was modulated separately. TLS2 and TLS3 (channels 2 and 3, respectively) served as interference channels for TLS1 (channel 1). Maximum OPA gain was achieved by aligning the SOPs of signals and pump by PC3, PC5, and PC8. Signals and pump were then combined by a 3-dB coupler and entered the DSF. The signal input powers into the DSF were maintained below $-18 \mathrm{dBm}$, while the input pump power was $18.23 \mathrm{dBm}(66.5 \mathrm{~mW})$. This ensured that the pump was undepleted and that low-signal-related nonlinear effects, such as cross-gain modulation (XGM) [10] and FWM, would be generated. The output spectrum of the DSF was observed with an optical spectrum analyzer (OSA). TBF2 selected the desired signal wavelength before detection by the optical-toelectrical $(\mathrm{O} / \mathrm{E})$ converter for performance measurements.

We first removed TBF1 in order to inject a broad EDFA ASE spectrum into the DPA and turned off all the signal channels. The shape of the ASE spectrum at the output of the DPA then provided an indication of the DPA gain spectrum. The experimental data for the parametrically amplified ASE spectrum is shown in Fig. 3(a). Its shape shows a 10-dB bandwidth of about $2 \mathrm{~nm}$ on each side of the pump, which can be used for amplifying transmission signals. The gain bandwidth is smaller than expected from theory (Fig. 1), which may be due to substantial longitudinal variations of $\lambda_{0}$ in this threesegment link. A nondestructive measurement of the optical fiber dispersion map [11], [12] would be essential for the design of a DPA scheme.

We then inserted TBF1 to efficiently suppress EDFA ASE and turned on the signals one by one. Three cases were examined:

1) only channel 1 on (single-channel transmission);

2) channels 1 and 3 on, in which case XGM effects were generated in the DPA; and

3) all three channels were on. 


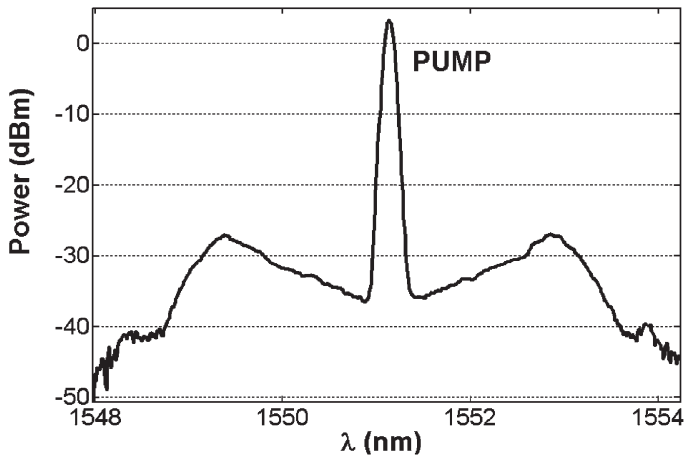

(a)

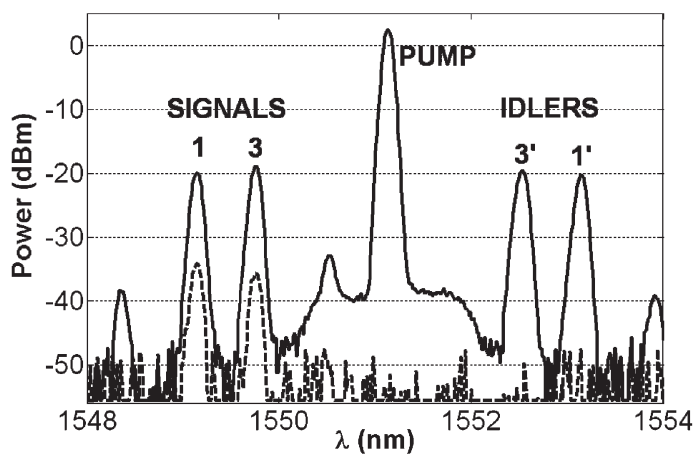

(c)

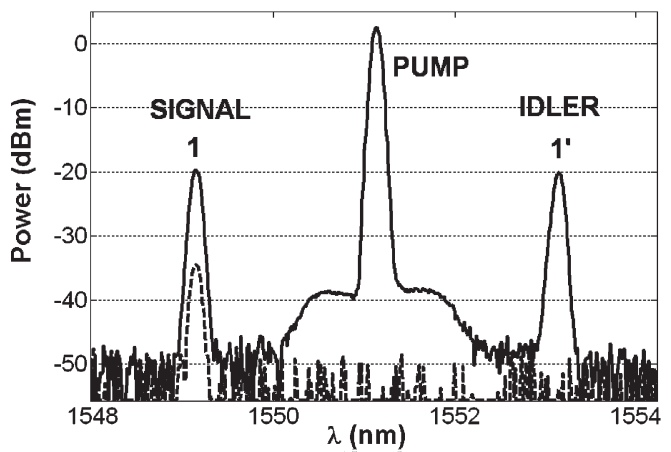

(b)

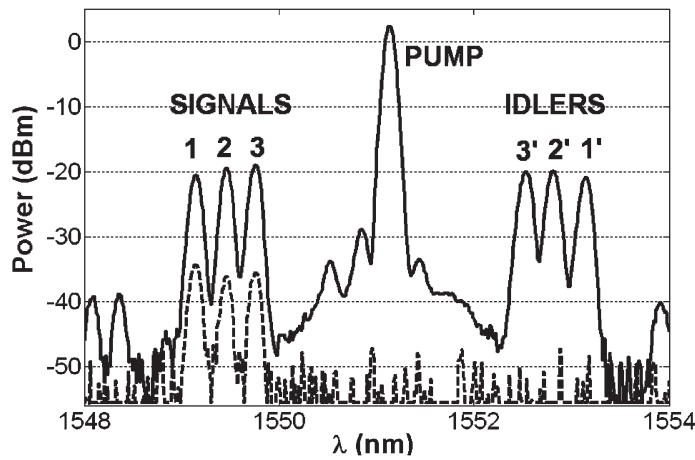

(d)

Fig. 3. (a) ASE output spectrum at the output of the distributed OPA with $18.23 \mathrm{dBm}$ of input pump power; spectrum at DPA output for (b) single-channel (channel 1 on), (c) two-channel (channels 1 and 3 on), and (d) three-channel (channels 1, 2, and 3 on) transmission.
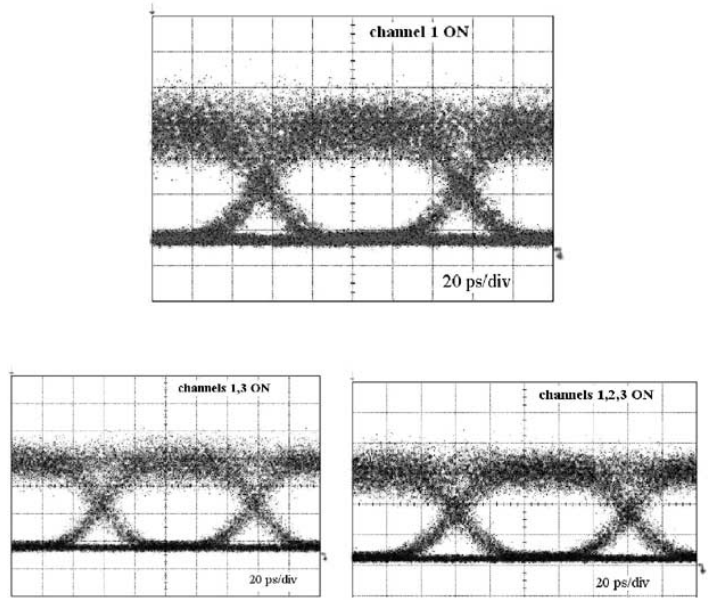

(a)

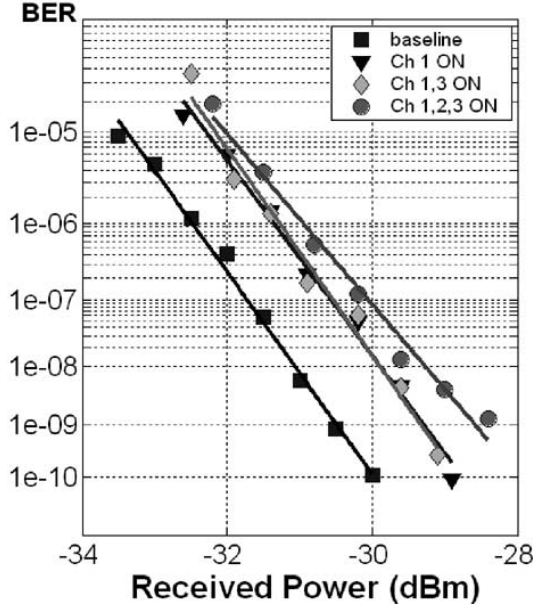

(b)

Fig. 4. (a) Eye patterns and (b) BER plots for received signal at $1549.14 \mathrm{~nm}$ after the DPA for the three cases examined and for the back-to-back configuration.

The choice of the channel wavelengths was such that channels 2 and 3 generated an FWM component at the wavelength of channel 1. Therefore, both XGM and FWM effects were generated on channel 1 in this case.

The powers of channels 1,2 , and 3 at the DSF input were $-18.74,-20.71$, and $-20 \mathrm{dBm}$, respectively. The corresponding ON-OFF signal gains were $14.4,16.9$, and $16.5 \mathrm{~dB}$. The gain is sufficient to compensate the transmission loss of the $75-\mathrm{km}$ DSF $(15.52 \mathrm{~dB})$, in agreement with the theoretical predictions. The optical spectra observed at the output of the DSF for these three cases are shown in Fig. 3(b)-(d), respectively, when pump was ON (solid lines) or OFF (dashed lines).

The amplified signal at $1549.14 \mathrm{~nm}$ (channel 1) was then selected by TBF2 and detected by an optical receiver. The eye patterns were displayed on an oscilloscope for the three cases discussed above. Examples are shown in Fig. 4(a); the clear opening indicates the high quality of the received signal. The bit error rate (BER) was also measured by varying the received power by means of variable optical attenuator (VOA1). BER plots are shown in Fig. 4(b), together with a reference baseline 


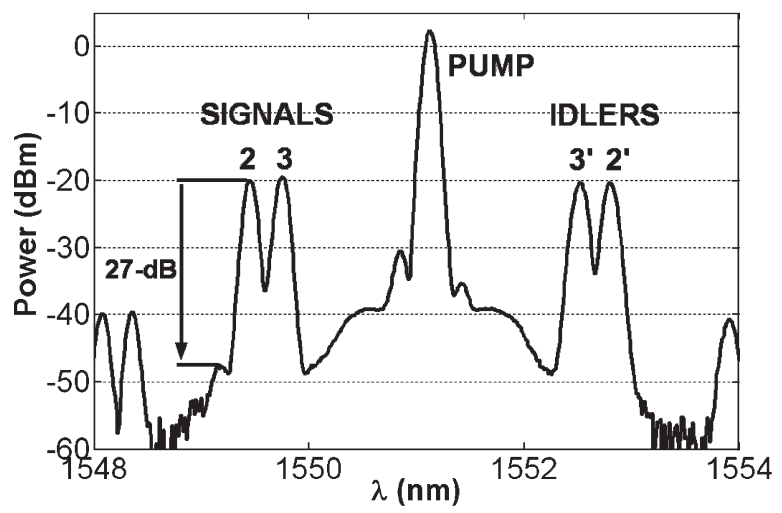

Fig. 5. Spectrum at the output of the distributed OPA when channels 2 and 3 are on. The FWM component due to the mixing of those channels falls at the channel 1 wavelength. It is measured to be $27 \mathrm{~dB}$ lower than the power level of the signals.

for the transmitter and receiver back to back (the baseline was taken with only channel 1 on, bypassing the DSF and directly connecting to the optical receiver). It can be seen that the insertion of the DPA led to a power penalty of the order of $1.2 \mathrm{~dB}$ [cases 1) and 2)], and $2.1 \mathrm{~dB}$ [case 3)]. The degradation in the signal quality for case 1) was mainly due to intensity noise transfer from pump to signal [13], and conversion of pump phase modulation to signal intensity modulation along the DSF [14], [15]. Both of these effects degraded the mark level of the signal eye. Another source of degradation was possibly due to SBS, which is generally more severe for a distributed OPA due to the long fiber (in our experiment the fiber effective length was $L_{\text {eff }} \approx 21 \mathrm{~km}$ ). Fig. 4(a) confirms that the eye opening was limited by excess noise in the mark level. The power penalty for case 2) was the same as that for case 1 ); the lower input signal power levels compared to the pump power guarantee substantially low XGM effects. The penalty for case 3) increased to $2.1 \mathrm{~dB}$. Fig. 5 shows the spectrum at the output of the DSF when only channels 2 and 3 were present. These channels created an FWM component at the wavelength of channel 1. This component was measured to be $27 \mathrm{~dB}$ below the power level of channel 1 . Therefore, the increased penalty when all three channels were present was mainly due to the imperfect filtering of interfering channel 2 at the receiver.

\section{COMPARISON WITH DRA}

The DPA scheme was then compared experimentally with DRA. Single-channel transmission was used for the comparison. Signal power at the input of the transmission fiber was kept at $-5 \mathrm{dBm}$ in both cases. The experimental setup for the DPA case was similar to Fig. 2. A pump power of $79 \mathrm{~mW}$ provided $14.1 \mathrm{~dB}$ of gain to the signal, about $1.4 \mathrm{~dB}$ less than the total loss of the DSF span. Increasing the pump-power level to completely compensate for the fiber loss degraded the quality of the signal substantially due to strong SBS effects. The output was then filtered, and the transmitted channel was detected by an optical receiver. The clear eye diagram shown in Fig. 6(a) indicates the high quality of the received signal. BER plots yielded a penalty of $1.2 \mathrm{~dB}$ at a BER level of $10^{-9}$ [Fig. 6(b)].
The experimental setup for the DRA case is shown in Fig. 7. Backward Raman pumping was used, since it is known to provide better noise performance than forward pumping [16]. The pump was a high-power fiber Raman laser. A pump power of $282 \mathrm{~mW}$ provided $14.1 \mathrm{~dB}$ of distributed gain to the signal. Its eye diagram showed a clear eye opening [Fig. 6(a)], and the BER plot indicates a $1.09-\mathrm{dB}$ penalty at a BER level of $10^{-9}$ [Fig. 6(b)]. When a forward-pumping scheme was tested for the same amount of gain, excess intensity noise transfer from the pump to the signal degraded the eye quality in the mark level considerably; therefore, a forward-Raman-pumping scheme was not considered in the comparison. Table I summarizes the experimental comparison results.

\section{Discussion}

Our experimental results have verified that it is indeed possible to transmit communication signals over a typical fiber link by DPA, with remarkably low pump power. Even though we did not take particular precautions to have a very low relative intensity noise (RIN) pump, and SBS was difficult to suppress, we obtained sufficient signal quality at the receiver for low-penalty reception. Additional channels did not cause large degradation due to nonlinear crosstalk. Direct comparison with a backward-pumped DRA system showed that the DPA required only about one third the pump power of the DRA which can be a considerable advantage.

Because the pump power $P_{0}$ required to make up for fiber loss is relatively modest (less than $100 \mathrm{~mW}$ ), the theoretical DPA gain bandwidth is fairly small, of the order of $10 \mathrm{~nm}$ or less. Furthermore, in practical fibers, the bandwidth depends on the $\lambda_{0}$ distribution along the fiber length. In our case, it is reduced from a theoretical value of about $10 \mathrm{~nm}$ (assuming constant $\lambda_{0}$ along the fiber span) to about $2 \mathrm{~nm}$ (for the actual three-spool of DSF span). This is much smaller than for DRA and so DPA will not compete with DRA, but could be operated to provide supplementary capacity.

An aspect that should to be taken into account is polarization dependence. The gain in the DRA case showed a dependence on the input signal polarization of less than $1 \mathrm{~dB}$, much less than the polarization dependence of the gain in the DPA case (of the order of $10 \mathrm{~dB}$ ). In the relatively polarization-independent DRA, only half the pump power contributes to signal amplification (that is, only $141 \mathrm{~mW}$ in the above experiment). Therefore, for a fair comparison, a polarization-independent parametric-amplification scheme [17] should have been employed. While such a scheme has not yet been demonstrated in long fibers, the fact that parametric amplification with two parallel pumps works well in a 25-km-long DSF [5] indicates that there is a good possibility that the same would be true with two orthogonal pumps. In such a case, three times as much pump power would be needed (i.e., $237 \mathrm{~mW}$ ). Nevertheless, our comparison confirmed that the DPA scheme is capable of performing similar to the DRA scheme, while using less pump power. These considerations would be particularly important in a multiple-segment link, where it would be required to use polarization-independent amplification to account for the random polarization of signals incident on downstream segments. 

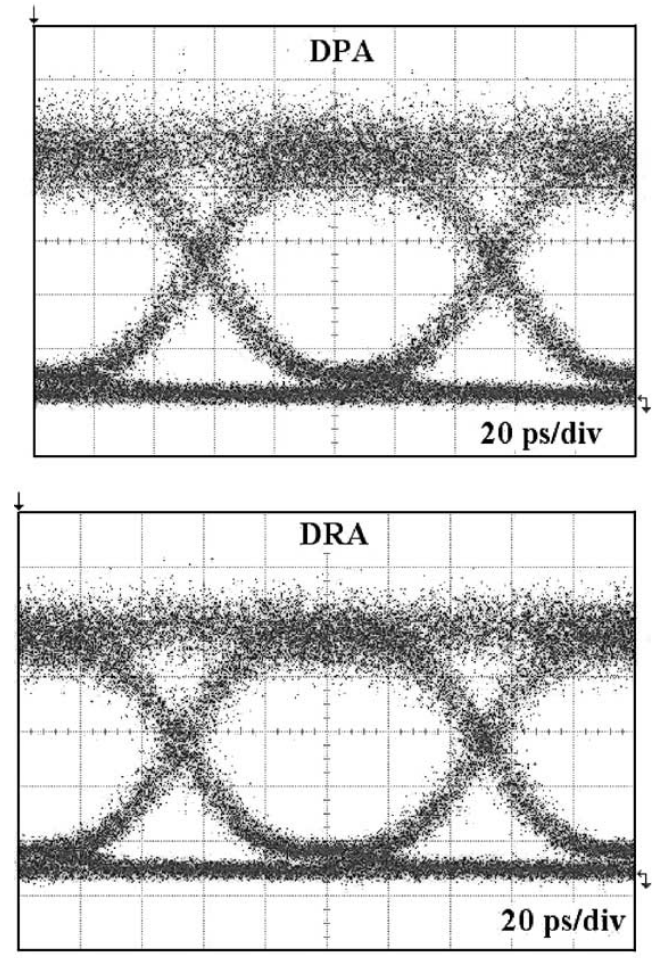

(a)

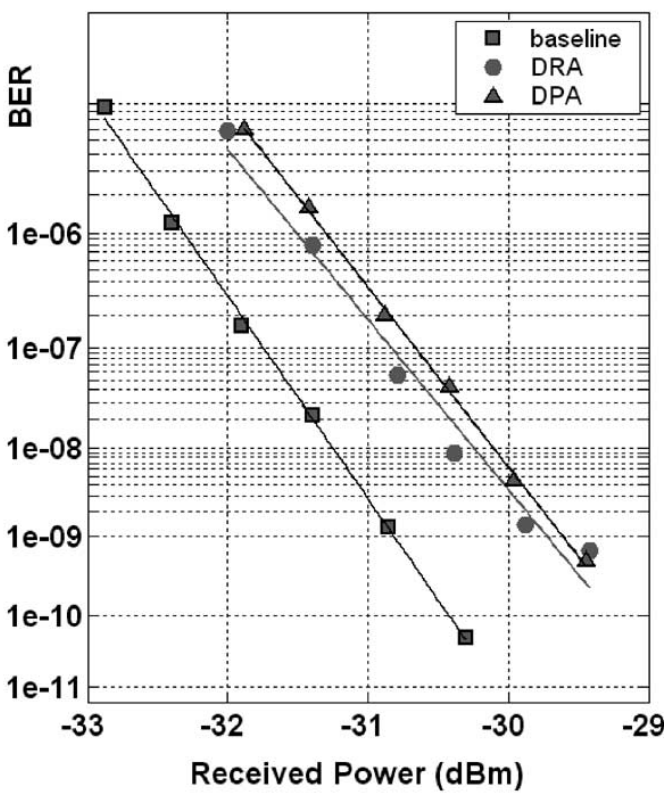

(b)

Fig. 6. (a) Eye diagrams for the DPA and DRA cases, and (b) corresponding BER performances. Penalties at BER $=10^{-9}$ are 1.2 and $1.09 \mathrm{~dB}$ for the DPA and DRA, respectively.

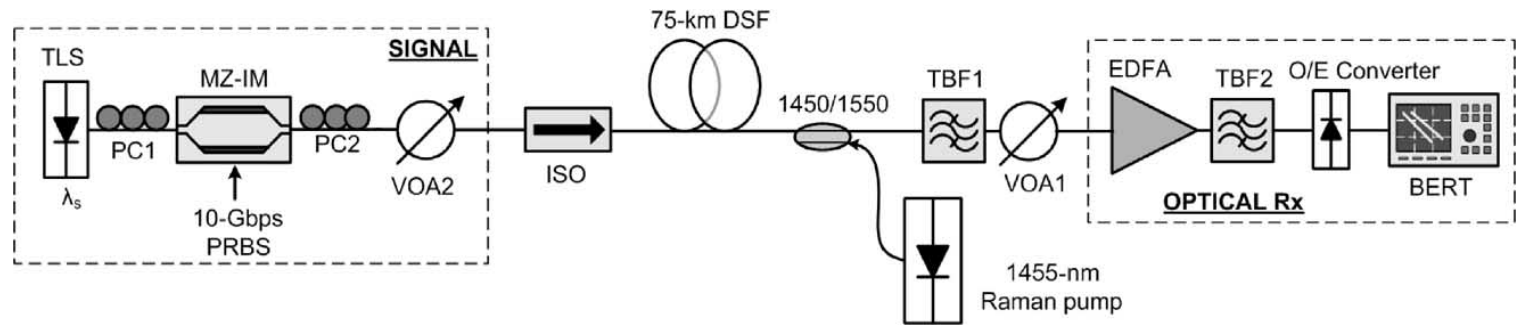

Fig. 7. Experimental setup for DRA.

An interesting aspect of DPA is the presence of the idler, which can potentially be used to improve system performance in two different ways:

1) Signal and idler carry the same information through their modulations. In principle, one could then have separate receivers for signal and idler and combine their electrical outputs. As a result, signal-to-noise ratio could improve by up to $3 \mathrm{~dB}$, as in a coherent detector using balanced detection.

2) The inverted spectrum of the idler offers opportunities for canceling some nonlinear effects in cascaded links, as has been done with discrete spectral inverters in mid-span spectral inversion (MSSI).

\section{CONCLUSION}

The authors have investigated, for the first time to the authors' knowledge, the use of DPA for transmitting communication signals over a typical fiber communication link
TABLE I

DPA-DRA COMPARISON

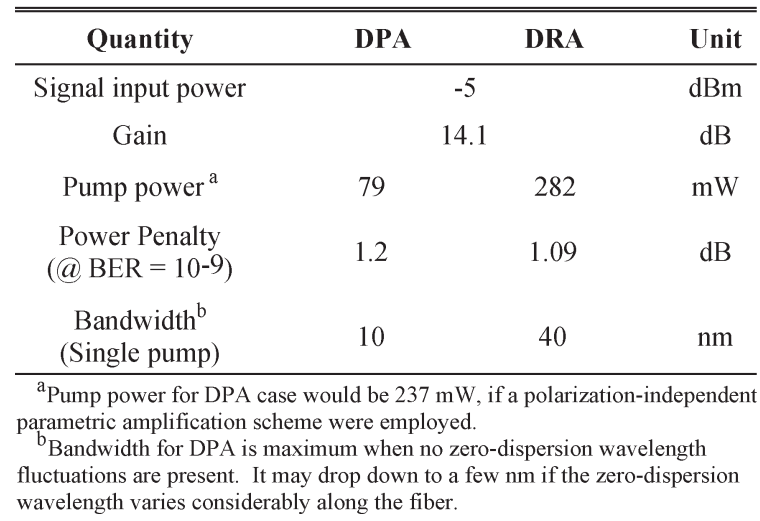

and have derived expressions for the gain in uniform lossy fibers in terms of hypergeometric functions. Theory predicts that loss can be compensated with a low pump power, typically below $100 \mathrm{~mW}$, and that bandwidths of the order of 
$10 \mathrm{~nm}$ could be obtained near the zero-dispersion wavelength. Experimentally, the authors have obtained low-penalty transmission of a $10-\mathrm{Gb} / \mathrm{s}$ communication signal along a $75-\mathrm{km}$ transmission fiber with only $66.5 \mathrm{~mW}$ of pump power; additional channels did not cause great degradation due to nonlinear crosstalk. A DRA scheme with similar performance required $282 \mathrm{~mW}$ of pump power.

DPA provides a novel means of signal amplification in communication networks, which may find practical applications for single- or multichannel systems.

\section{APPENDIX A}

We obtain the Wronskian of the Whittaker functions by using their relations with the Kummer functions $M(a, b, Z)$ and $U(a, b, Z)$, for which the Wronskian is known. These relations are

$$
\begin{aligned}
& M_{\kappa, \mu}(Z)=\mathrm{e}^{-\frac{Z}{2}} Z^{\frac{b}{2}} M(a, b, Z) \\
& W_{\kappa, \mu}(Z)=\mathrm{e}^{-\frac{Z}{2}} Z^{\frac{b}{2}} U(a, b, Z)
\end{aligned}
$$

where $a=(1 / 2+\mu-\kappa)$ and $b=(1+2 \mu)$. From these, we obtain relations between the derivatives with respect to $Z$ (denoted in this context by a prime)

$$
\begin{aligned}
M_{\kappa, \mu}^{\prime}(Z)= & \mathrm{e}^{-\frac{Z}{2}} Z^{\frac{b}{2}} M^{\prime}(a, b, Z) \\
& +\frac{1}{2}\left(\frac{b}{Z}-1\right) M_{\kappa, \mu}(Z) \\
W_{\kappa, \mu}^{\prime}(Z)= & \mathrm{e}^{-\frac{Z}{2}} Z^{\frac{b}{2}} U^{\prime}(a, b, Z) \\
& +\frac{1}{2}\left(\frac{b}{Z}-1\right) W_{\kappa, \mu}(Z) .
\end{aligned}
$$

Since the Wronskian of the Kummer functions is

$$
\begin{aligned}
W_{\mathrm{Kum}}(Z) & =M(a, b, Z) U^{\prime}(a, b, Z)-U(a, b, Z) M^{\prime}(a, b, Z) \\
& =-\frac{\mathrm{e}^{Z} Z^{-b} \Gamma(b)}{\Gamma(a)}
\end{aligned}
$$

we then find that

$$
\begin{aligned}
W_{\text {Whit }}(Z) & =\mathrm{e}^{-Z} Z^{b} W_{\text {Kum }}(Z) \\
& =\mathrm{e}^{-Z} Z^{b}\left[-\frac{\mathrm{e}^{Z} Z^{-b} \Gamma(b)}{\Gamma(a)}\right] \\
& =-\frac{\Gamma(b)}{\Gamma(a)} .
\end{aligned}
$$

\section{APPENDIX B}

The propagation equations for the signal and the idler in a lossy OPA are given by (1), or

$$
\frac{\mathrm{d} A_{k}}{\mathrm{~d} z}=-p A_{k}+r \exp \left(\int_{\xi=0}^{z} q \mathrm{~d} \xi\right)\left(A_{7-k}\right)^{*}, \quad k=3,4 .
$$

Defining the power of the $k$ th wave at $z$ as $P_{k}=A_{k} A_{k}^{*}$, we obtain

$$
\begin{aligned}
\frac{\mathrm{d} P_{k}}{\mathrm{~d} z}=-\alpha P_{k}+r \exp & \left(\int_{\xi=0}^{z} q \mathrm{~d} \xi\right) \\
& \times A_{k}^{*}\left(A_{7-k}\right)^{*}+\text { c.c. } \quad k=3,4 .
\end{aligned}
$$

By subtracting these two equations, we obtain

$$
\frac{\mathrm{d}}{\mathrm{d} z}\left(P_{3}-P_{4}\right)=-\alpha\left(P_{3}-P_{4}\right)
$$

Equation (A9) yields, by integration, $P_{3}-P_{4}=\left[P_{3}(0)-\right.$ $\left.P_{4}(0)\right] \exp (-\alpha z)$. Assuming that $P_{4}(0)=0$, and dividing both sides by $P_{3}(0)$, we obtain

$$
G_{\mathrm{s}}=G_{\mathrm{i}}+\mathrm{e}^{-\alpha z}
$$

This result has a simple physical interpretation, which can be obtained by investigating what factors influence the power imbalance $P_{3}-P_{4}$. Since signal and idler each gain one photon at the same time, photon emission does not affect $P_{3}-P_{4}$. Thus, the only way that a power imbalance can exist within the fiber is if one exists at the fiber input. Then, because the two waves decay at the same rate $\alpha$, so does $P_{3}-P_{4}$. This is expressed mathematically by (A9), which then leads to (A10).

\section{ACKNOWLEDGMENT}

The authors would like to thank the Optoelectronic Industry Development Association (OIDA) for providing the high-power EDFA and filter, and Sprint Advanced Technology Laboratories for the loan of equipment.

\section{REFERENCES}

[1] M. E. Marhic, K. K.-Y. Wong, G. Kalogerakis, and L. G. Kazovsky, "Toward practical fiber optical parametric amplifiers and oscillators," Optics Photonics News, vol. 15, no. 9, pp. 20-25, Sep. 2004.

[2] N. Henmi, Y. Aoki, T. Ogata, T. Saito, and S. Nakaya, "A new design arrangement of transmission fiber dispersion for suppressing nonlinear degradation in long-distance optical transmission systems with optical repeater amplifiers," J. Lightw. Technol., vol. 11, no. 10, pp. 1615-1621, Oct. 1993.

[3] G. Bosco, A. Carena, V. Curri, R. Gaudino, P. Poggiolini, and S. Benedetto, "A novel analytical approach to the evaluation of the impact of fiber parametric gain on the bit error rate," IEEE Trans. Commun., vol. 49, no. 12 , pp. 2154-2163, Dec. 2001.

[4] P. Serena, A. Orlandini, and A. Bononi, "A parametric gain approach to DPSK performance evaluation in presence of nonlinear phase noise," presented at the Eur. Conf. Optical Communication (ECOC), Stockholm, Sweden, Sep. 2004, Paper We4.P.124.

[5] J. M. Chavez Boggio, S. Tenenbaum, and H. L. Fragnito, "Amplification of broadband noise pumped by two lasers in optical fibers," J. Opt. Soc. Amer. B, vol. 18, no. 10, pp. 1428-1435, Oct. 2001.

[6] M. E. Marhic, K. K.-Y. Wong, and L. G. Kazovsky, "Fiber optical parametric amplifiers with linearly- or circularly-polarized waves," J. Opt. Soc. Amer. B, vol. 20, no. 12, pp. 2425-2433, Dec. 2003.

[7] M. Abramowitz and I. A. Stegun, Handbook of Mathematical Functions. New York: Dover, 1972.

[8] M. E. Marhic, Y. Park, F. S. Yang, and L. G. Kazovsky, "Broadband fiber optical parametric amplifiers and wavelength converters with lowripple Chebyshev gain spectra," Opt. Lett., vol. 21, no. 17, pp. 1354-1356, Sep. 1996. 
[9] K. K. Y. Wong, M. E. Marhic, K. Uesaka, and L. G. Kazovsky, "Polarization-independent two-pump fiber optical parametric amplifier," IEEE Photon. Technol. Lett., vol. 14, no. 7, pp. 911-913, Jul. 2002.

[10] T. Torounidis, H. Sunnerud, P. O. Hedekvist, and P. A. Andrekson, "Amplification of WDM signals in fiber-based optical parametric amplifiers," IEEE Photon. Technol. Lett., vol. 15, no. 8, pp. 1061-1063, Aug. 2003.

[11] M. Eiselt, R. M. Jopson, and R. H. Stolen, "Nondestructive positionresolved measurement of the zero-dispersion wavelength in an optical fiber," J. Lightw. Technol., vol. 15, no. 1, pp. 135-143, Jan. 1997.

[12] M. Artiglia, R. Caponi, E. Grazioli, A. Pagano, A. Panella, M. Potenza, E. Riccardi, and B. Sordo, "Measurement of the dispersion map of installed G.653 fiber links using four-wave mixing," presented at the Optical Fiber Communications Conf. (OFC), San Jose, CA, 1998, Paper WM2

[13] M. E. Marhic, G. Kalogerakis, K. K.-Y. Wong, and L. G. Kazovsky, "Pump-to-signal transfer of low-frequency intensity modulation in fiber optical parametric amplifiers," J. Lightw. Technol., vol. 23, no. 3, pp. 1049-1055, Mar. 2005.

[14] M. E. Marhic, G. Kalogerakis, and L. G. Kazovsky, "Pump FM to signal IM conversion in fiber OPAs," presented at the OptoElectoronics and Communications Conf./3rd Int. Conf. Optical Internet (OECC/COIN), Yokohama, Japan, Jul. 2004, Paper 13P-87.

[15] A. Mussot, A. Durecu-Legrand, E. Lantz, C. Simonneau, D. Bayart, H. Maillotte, and T. Sylvestre, "Impact of pump phase modulation on the gain of fiber optical parametric amplifier," IEEE Photon. Technol. Lett., vol. 16, no. 5, pp. 1289-1291, May 2004.

[16] C. R. S. Fludger, V. Handerek, and R. J. Mears, "Pump to signal RIN transfer in Raman fiber amplifiers," J. Lightw. Technol., vol. 19, no. 8, pp. 1140-1148, Aug. 2001.

[17] K. K.-Y. Wong, M. E. Marhic, K. Uesaka, and L. G. Kazovsky, "Polarization-independent one-pump fiber-optical parametric amplifier," IEEE Photon. Technol. Lett., vol. 14, no. 11, pp. 1506-1508, Nov. 2004.

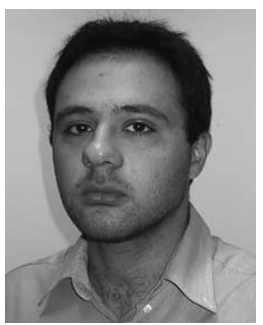

Georgios Kalogerakis (S'02) received the B.E. degree in electrical and computer engineering from the National Technical University of Athens, Greece, in 2001 and the M.S. degree in electrical engineering from Stanford University, Stanford, CA, in June 2003. He is currently working towards the Ph.D. degree in electrical engineering at Stanford University.

He is a member of the Photonics and Networking Research Laboratory at Stanford University. His research is focused on fiber nonlinearity, fiber optical parametric amplifiers, and high-speed optical transmission systems.

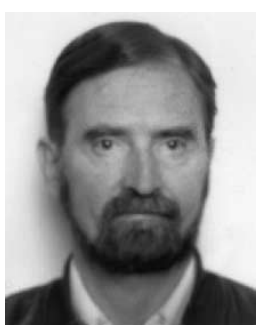

Michel E. Marhic (M'79-SM'89) received the Diplome D'Ingenieur from Ecole Superieure D'Electricite, France, the M.S. degree from Case Western Reserve University, Cleveland, $\mathrm{OH}$, and the Ph.D. degree from University of California, Los Angeles, all in electrical engineering.

$\mathrm{He}$ was on the faculty of the Department of Electrical Engineering at Northwestern University (1974-1998), and on sabbatical leaves at University of Southern California, Los Angeles (1979-1980) and Stanford University, Stanford, CA (1984-1985, and 1993-1994). He is currently a Consulting Professor in the Department of Electrical Engineering at Stanford University. He (co)-founded Holicon, Holographic Industries, and OPAL Laboratories. Over the past 25 years, his research has been in several areas of applied optics, including nonlinear interactions in plasmas; optical fiber measurements; hollow infrared waveguides; holography and phase conjugation; and fiber networks. Over the past ten years, emphasis has been on optical communication systems and on nonlinear optical interactions in fibers. He is the author or coauthor of over 240 journal and conference papers, and had eight patents awarded.

Dr. Marhic is a Member of Optical Society of America (OSA), and eminent Member of Tau Beta Pi. He was a recipient of the Ameritech Research Professorship from the Institute for Modern Communications in 1990-1991.

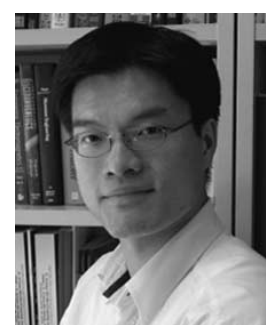

Kenneth Kin-Yip Wong (S'00-M'03) received the combined B.E. (first-class honors with medal award) degree in electrical engineering, the B.S. degree in physics from the University of Queensland, Brisbane, Australia, in 1997, and the M.S. and $\mathrm{Ph} . \mathrm{D}$. degrees in electrical engineering from Stanford University, Stanford, CA, in 1998 and 2003, respectively.

His research field included dense wavelength division multiplexing (DWDM) systems, subcarrier multiplexing (SCM) optical systems, fiber nonlinearity, fiber optical parametric amplifiers, and photonic crystal fibers. He is the author or coauthor of over 50 journal and conference papers. He worked in Hewlett-Packard Laboratories as Research Engineer and contributed to projects including parallel optics and vertical-cavity surface-emitting laser (VCSEL) in 1998-1999. He was an Independent Consultant in Innovation CORE (A Sumitomo Electric Company), CA, in 2004. He is currently an Assistant Professor in the Department of Electrical and Electronic Engineering in the University of Hong Kong, Pokfulam, Hong Kong.

Dr. Wong is a Member of the International Society for Optical Engineers (SPIE) and the IEEE Lasers \& Electro-Optics Society (LEOS) and was a Member of the Photonics and Networking Research Laboratory at Stanford University. He was the recipient of OSA New Focus Student Award and IEEE/LEOS Graduate Student Fellowship, both in 2003. He is reviewer for Optics Letters, JOSA B, Optics Express, IEEE PHOTONICS TECHNOLOGY LetTers, Journal OF LightwaVe TeChNOLOGY, and Optics Communications.

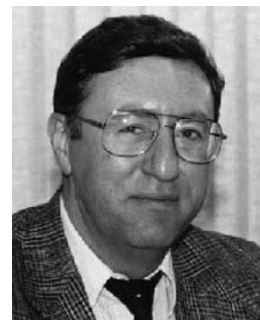

Leonid G. Kazovsky (M'80-SM'83-F'91) has been a Professor of Electrical Engineering at Stanford University, Stanford, CA, since 1990. After joining Stanford, he founded Optical Communication Research Laboratory (OCRL). He has led OCRL since then. Prior to joining Stanford, he was with Bellcore (now Telcordia) doing research on wavelength-division-multiplexing (WDM) and highspeed and coherent optical fiber communication systems. While on Bellcore assignments or Stanford sabbaticals, he worked at the Heinrich-Hertz Institute, Berlin, Germany; Hewlett-Packard Research Laboratories, Bristol, U.K. and Technical University of Eindhoven, The Netherlands. Through research contracts, consulting engagements, and other arrangements, he worked with many industrial companies and U.S. Government agencies including Sprint, DEC, GTE, AT\&T, IVP, Lucent, Hitachi, KDD, Furukawa, Fujitsu, Optivision, and Perimeter on the industrial side; and the National Science Foundation (NSF), Defense Advanced Research Agency (DARPA), Air Force, Navy, Army, and BMDO on the government side. In 1998 to 1999 , he took a one-year leave from Stanford University and launched a start-up company now known as Alidian Networks. He serves on the Board of Directors of that company. $\mathrm{He}$ authored or coauthored two books, some 150 journal technical papers, and a similar amount of conference papers.

Prof. Kazovsky is a Fellow of the Optical Society of America (OSA). He serves or served on Editorial Boards of leading journals (IEEE TRANS ACTIONS on COMmunications, IEEE Photonics TECHNOLOGY LetTERs, Wireless Networks) and on Program Committees of leading conferences (OFC, CLEO, LEOS, SPIE, and GLOBECOM). He also served as a reviewer for various IEEE and IEE transactions, proceedings, and journals; funding agencies (NSF, OFC, ERC, NRC, etc.), and publishers (Wiley, MacMillan, etc.). 